\title{
Uveal metastatic disease: Current and new treatment options (Review)
}

\author{
GIAN PAOLO GIULIARI ${ }^{1,2}$ and AMA SADAKA ${ }^{3}$ \\ ${ }^{1}$ Centro de Cirugía Oftalmológica (CECOF), Caracas; ${ }^{2}$ Department of Ophthalmology, Domingo Luciani Hospital, \\ Caracas, Venezuela; ${ }^{3}$ Department of Ophthalmology, Massachusetts Eye and Ear Infirmary, Boston, MA, USA
}

Received July 15, 2011; Accepted August 22, 2011

DOI: $10.3892 /$ or.2011.1563

\begin{abstract}
Choroidal metastasis represents the most common form of intraocular malignancies. It may occur in up to $10 \%$ of patients with systemic metastasis with almost half of the patients developing central nervous system disease. The most common primary sites of ocular metastasis are breast cancer in women and lung cancer in men. In most cases, these lesions tend to be asymptomatic and are not evaluated by an ophthalmologist. The diagnosis is generally made by the history of present or prior malignancies and an ophthalmological examination with slit-lamp biomicroscopy and indirect ophthalmoscopy. As with other malignancies, management may vary with each patient. Small tumors, that do not compromise the vision and that have responded previously to systemic treatment, may be closely observed. For larger lesions and for symptomatic ones, external beam radiation offers an excellent alternative to save the eye and stabilize vision. Bevacizumab (Avastin), a potent monoclonal antibody that has also been employed for the treatment of ocular vaso-proliferative diseases, has been used in the treatment of choroidal metastasis and has shown promising results.
\end{abstract}

\section{Contents}

1. Introduction

2. Patient characteristics

3. Symptoms and clinical characteristics

4. Diagnosis

5. Treatment

6. Conclusions

Correspondence to: Dr Gian Paolo Giuliari, Centro de Cirugía Oftalmológica (CECOF), Av. Francisco de Miranda, Torre Cavendes, Piso 6, Ofic. 6-04 Altamira, Caracas, Venezuela

E-mail: gpgiuliari@gmail.com

Key words: ocular malignancy, uveal metastasis, choroidal metastasis, ocular masquerade syndrome, vascular endothelial growth factor, bevacizumab

\section{Introduction}

Technological ophthalmic advances have led to an increase in the reported frequency of uveal metastasis (1). Albert et al found that $2 \%$ of 213 patients with systemic cancer had uveal metastasis (2). At present, choroidal metastasis is accepted to be the most common intraocular malignancy $(1,3)$. However, in an ocular oncology practice setting, it is not encountered as frequently as primary uveal melanoma, possibly due to the fact that many of these patients with metastatic systemic disease have asymptomatic ocular lesions and thus are not brought to the attention of the ophthalmologist (2,4,5-7).

Given its very rich blood supply, the uvea is one of the most favored sites in the body for the seeding of tumor cells and the development of metastasis $(2,8)$. In up to $90 \%$ of ocular metastasis cases, the posterior aspect of the uvea (choroid) is affected, with the remaining $10 \%$ arising from the iris and/or ciliary body (9). Metastasis to other ocular sites such as the retina, optic nerve and vitreous, although described, remains uncommon $(10,11)$.

The most common types of cancer that metastasize to the eye are carcinomas, however, sarcomas and melanomas may also metastasize to the eye $(12,13)$. The prevalence of uveal metastasis from all forms of carcinomas varies from 2.3 to $9.2 \%$ in the published series with breast cancer being the most common primary site followed by lung cancer $(2,5,9)$. Other sites of primary malignancies are the kidney, thyroid, prostate, pancreas, testis and other organs (14-19).

\section{Patient characteristics}

Although the majority of patients have a known history of carcinoma at the time of the diagnosis of uveal disease $(9,20,21)$, Shields et al reported no known history of cancer in $34 \%$ of their patients at the time of diagnosis of the uveal metastasis, with up to $10 \%$ of the patients remaining with an occult primary cancer (9). Upon diagnosis of uveal metastasis, more than half of the patients had associated systemic metastasis with the lungs being the most frequent site with concomitant involvement, followed by the bone, liver and central nervous system $(20,22)$. Mewis and Young found that 30 out of their 67 patients with choroidal metastasis also developed central nervous system disease (23). Moreover, Wiegel et al described the presence of lung and brain metastasis as the 


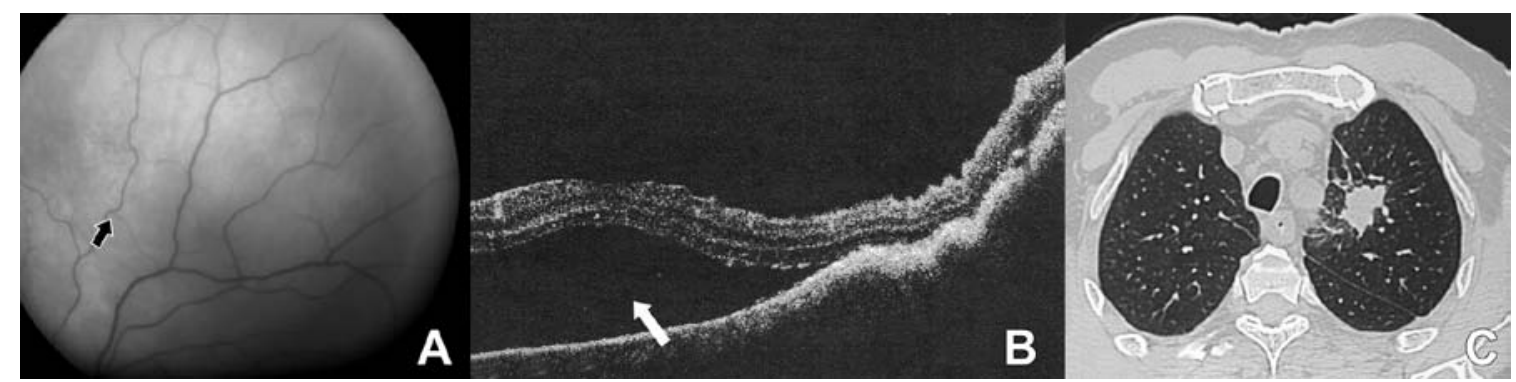

Figure 1. A 69-year-old man with no known history of cancer presenting with decreased visual acuity. Given the ophthalmic findings observed and his past history of smoking, he was diagnosed with a choroidal tumor possibly secondary to metastatic lung cancer and was referred to the oncology department where the diagnosis was later confirmed. (A) Fundus image of the left eye showing an amelanotic choroidal lesion superior to the macula (arrow). (B) Optical coherent tomography demonstrating subretinal fluid in the inferior aspect of the lesion that compromises the fovea (arrow). (C) CT scan of the thorax demonstrating a solid lesion with irregular borders in the superior third of the left lung.

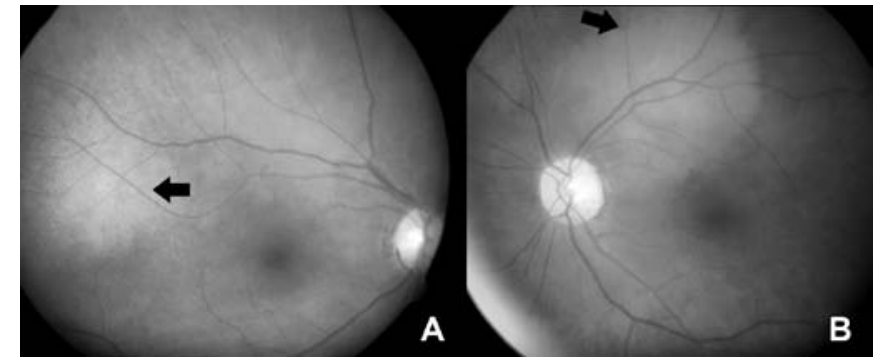

Figure 2. Fundus image of a 52-year-old woman with metastatic breast carcinoma. (A) Right eye showing a choroidal lesion temporal to the macula (arrow). (B) Left eye showing juxtapapillary choroidal metastasis (arrow).

only significant risk factors for the development of choroidal disease in patients with breast carcinomas with an incidence of $11 \%$ in the presence of these factors as compared to $5 \%$ in their absence (5). Kreusel et al found that metastasis in more than one organ is a significant risk factor for choroidal metastasis in patients with lung cancer (7). The prognosis following diagnosis of uveal metastasis is poor, with a median survival of 6-9 months $(7,22-24)$.

\section{Symptoms and clinical characteristics}

Symptoms of ocular metastasis may vary greatly depending upon the location and characteristics of the lesion. Although most of the patients remain asymptomatic (5-7,25), whenever symptoms appear the most common complaint is blurred vision $(2,5,7,22,26)$. In cases of choroidal metastasis, the blurring may be secondary to macular involvement or the presence of subretinal fluid that may compromise the fovea (9). Moreover, in cases of iris and/or ciliary body lesions, the decreased visual acuity is usually secondary to seeding to the anterior chamber or the development of secondary cataracts as these lesions tend to produce inflammatory signs more often than choroidal lesions (27). Other symptoms include visual field scotomas, floaters, metamorphopsia and photopsia $(22,26)$. In cases of anterior segment metastasis, a visible mass can be noted and the patient may notice a red eye (27).

As mentioned above, most of the uveal lesions involve the choroid, followed by the iris and the ciliary body $(9,20)$. Choroidal lesions are more frequently located in the area

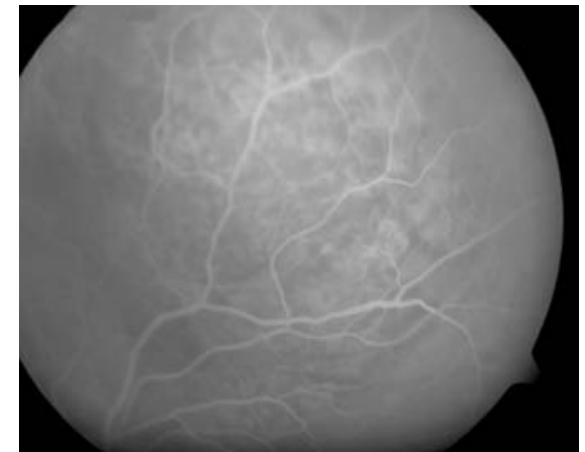

Figure 3. Retinal fluorescein angiography of a choroidal metastatic lesion in a 69 -year-old man with a metastatic lung cancer. Note the hyperfluorescence of the mass in the late venous phase.

between the macula and the equator (9). They usually appear as creamy or yellow choroidal lesions that may be associated with subretinal fluid (Fig. 1). Choroidal lesions secondary to bronchial carcinoid tumors, renal cell carcinoma and thyroid cancer may often present with an orange color (9). Some lesions may show the so-called 'leopard skin' appearance due to the brown pigmentation on their surface secondary to lipofuscincontaining macrophages. In up to $1 / 3$ of the cases, the affected eye may present with more than one focus of metastasis and up to $50 \%$ of patients may have bilateral involvement $(5,20,23,26)$ (Fig. 2).

\section{Diagnosis}

Diagnosis is usually made by the clinical findings noted during ophthalmic examination with slit-lamp biomicroscopy and indirect ophthalmoscopy, along with the history of a systemic malignancy. In cases of doubt, especially in the absence of the diagnosis of a primary malignant site, the differential diagnosis should include other lesions such as granulomas from tuberculosis or sarcoidosis, amelanotic uveal melanomas and lymphomas (28-31). Ancillary testing with retinal fluorescein angiography may show hyperfluorescence of the mass in the late venous phase. Ocular ultrasound usually reveals a medium to high internal reflectivity. This test may aid in the diagnosis and even in management and follow-up of the response of these 


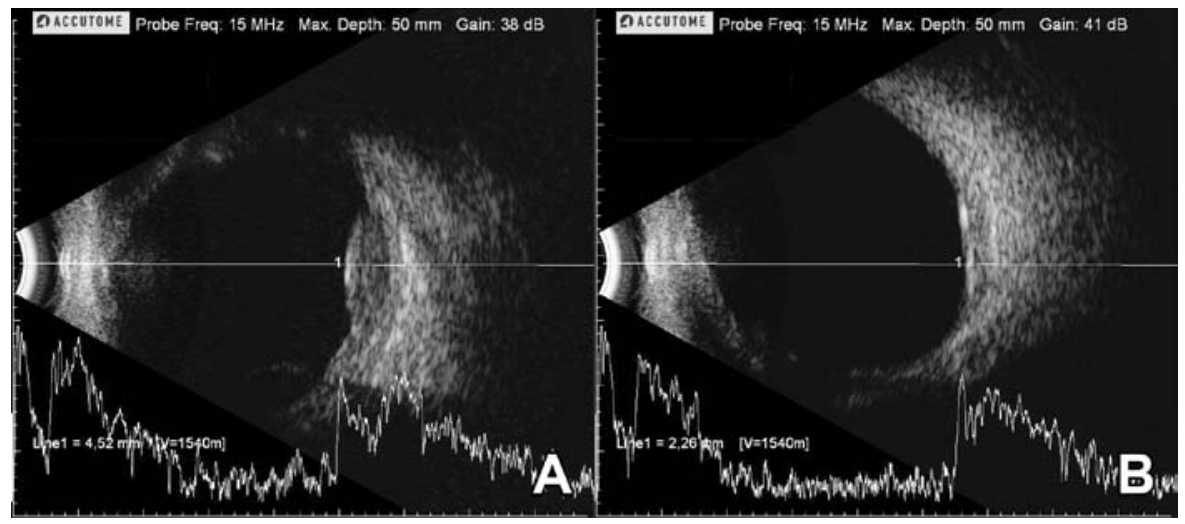

Figure 4. Ultrasound B and A mode of a 55-year-old woman with choroidal metastasis secondary to breast carcinoma. (A) Note the choroidal lesion with a height of $4.2 \mathrm{~mm}$ and a medium to high internal reflectivity. (B) Ultrasound of the same patient 6 weeks after treatment with $30 \mathrm{~Gy}$ over 10 days $(3 \mathrm{~Gy}$ each session) administered with external beam radiation. Note the significant decrease in the lesion height.

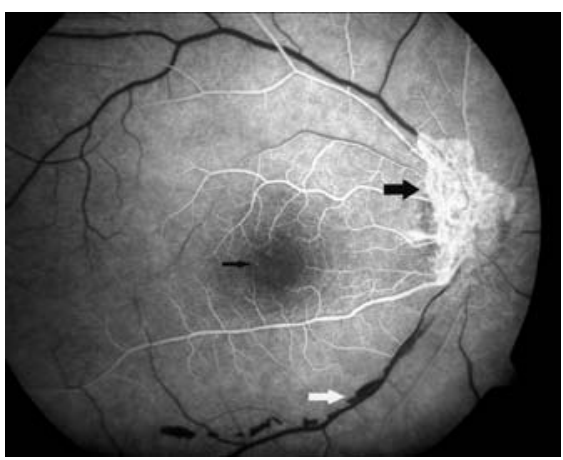

Figure 5. Early phase of a retinal angiogram of the right eye in a 42-year-old woman treated with external beam radiation due to an optic nerve meningioma. Note the active neo-vessels in the optic nerve head (thick black arrow) as well as the microaneurysms around the avascular zone of the fovea (thin black arrow) and the pre-retinal hemorrhages at the level of the inferior temporal vascular arcade (white arrow).

lesions to treatment (Fig. 3). A systemic evaluation by a multidisciplinary team is pivotal in the diagnosis and management of these patients, particularly in those cases with an occult primary site and the presence of rapid lesion growth which is characteristic of metastatic disease (9). In defined circumstances, a fine-needle biopsy can be performed in an effort to aid in the diagnosis and to establish the primary malignant site. However, in some cases, the uveal lesion is poorly differentiated making it challenging to determine the primary site and thus requiring immunohistochemical studies (24).

\section{Treatment}

Treatment of patients with choroidal metastasis is challenging and requires a multidisciplinary team that involves an ophthalmologist, oncologist, a radiation oncologist and other members of the health-care group. More than half of the patients have another metastatic focus at the time the ocular lesion is found (9). Restaging of the disease should be carried out at the moment of a newly diagnosed ocular lesion and early treatment should be initiated in an effort to avoid significant vision loss. Treatment options include radiation, laser, chemotherapy, anti-vascular endothelial growth factor (VEGF) and enucleation of the eye.

Radiation. The treatment of choice remains external beam radiation $(22,32)$. As other forms of radiation, it induces damage of the DNA of the rapidly growing tumor cells. Radiation has also been used for the treatment of other intraocular malignancies such as uveal melanomas, retinoblastomas and lymphomas $(31,33,34)$. Radiation dose varies greatly but it is usually lower than the dose required for uveal melanomas thus decreasing the complications of radiation such as radiation retinopathy $(4,20,35)$. External beam radiotherapy usually requires 3-4 weeks of daily or inter-daily radiation. This technique has proven to be effective in reducing the lesion size and improving or stabilizing visual acuity $(21,22,36)$ (Fig. 4).

Other forms of radiation employed include proton beam therapy that is usually given as fractionated or as a single dose. This technique produces significant tumor regression and thus stabilizes the vision (37). Adversely, this modality is not currently available in several countries and centers as it carries the need for expensive equipment. Plaque brachytherapy that has also been used in the treatment of uveal melanoma, consists of the application of an episcleral plaque that conforms to the curvature of the sclera and contains the radioactive material along its inner surface (38-40). Brachytherapy requires two surgical procedures - one for the insertion of the plaque and a second one for its removal. The advantage of brachytherapy over other forms of radiation includes a more precise and targeted radiation delivery to the tumor site thus decreasing the development of radiation complications (35-41) (Fig. 5).

Laser treatment. Several types of lasers have been shown to provide beneficial results in the treatment of uveal metastasis. One of these techniques is transpupillary thermotherapy (TTT) which consists of delivering heat to the choroid and the retinal pigment epithelium through the pupil using a modified diode laser that produces tumor necrosis (42). Laser photocoagulation with the use of argon or krypton is another modality that was shown to be effective with no ocular complications in 10 patients after a follow-up of 4-30 months (43). Photodynamic therapy (PDT) is a 2-step process in which an intravenous-infusion of verteporfin is followed (typically 
15 min later) by irradiance with a $689-\mathrm{nm}$ laser for approximately $83 \mathrm{sec}$. Verteporfin binds to low density lipoproteins in the plasma that are then preferentially bound by choroidal neovascular membranes. Application of laser energy results in the formation of toxic oxygen species that induce thrombosis of choroidal neovessels, which have shown efficacy in the treatment of carcinoid tumors (44).

Chemotherapy. Chemotherapy depends upon the type of primary cancer. Several classes of cytotoxic agents have been employed with promising results. The mechanism of action and associated toxicities depend on the type of agent used. Most of the available data on the use of chemotherapeutic agents for the treatment of uveal metastases are limited to case reports $(20,21,23,45)$. The use of newer therapeutic agents, in particular, has caused tumor size reduction and even complete regression in some cases $(46,47)$.

Anti-vascular endothelial growth factor-targeted treatment. Bevacizumab (Avastin, Genentech) is a potent monoclonal antibody that blocks all VEGF-A isoforms. It was the first anti-VEGF therapy approved by the FDA for the treatment of colorectal, breast and lung cancer (48). Based on the fact that bevacizumab has been shown to benefit patients with different type of cancers, several trials were initiated to test its efficacy in treating metastatic disease including choroidal metastasis. Kim et al showed resolution of a choroidal metastatic lesion from a non-small cell cancer and improvement of visual acuity after intravitreal bevacizumab $(2.5 \mathrm{mg})$ injections in combination with oral erlotinib (49). Amselem et al reported the case of a 57-year-old woman with stage IV non-estrogen-sensitive breast carcinoma with bone and lung metastasis that received treatment with oxaliplatin and vinorelbine who presented with a solitary and elevated choroidal metastatic lesion in the right eye. She was offered treatment with an intravitreal injection of $4 \mathrm{mg}$ of bevacizumab. Her visual acuity improved and the choroidal lesion decreased in size 3 weeks after the injection (50). Kuo et al reported the case of a 65 -year-old woman with colorectal adenocarcinoma that underwent 6 months of chemotherapy with fluorouracil, leucovorin calcium and oxaliplatin. After treatment, the carcinoembryonic antigen levels dropped to within normal range and the treatment was suspended. Two years later, the patient developed decreased visual acuity in the left eye. Uppon examination, a large and elevated, orange choroidal metastasic lesion was noted that encouraged giving an intravitreal injection of bevacizumab $(1.25 \mathrm{mg})$. Visual acuity improvement was noted 5 days after the injection (51). Lin et al found similar results after offering an intravitreal injection of $4 \mathrm{mg}$ of bevacizumab to a 43-year-old man with a colon adenocarcinoma who developed bone and choroidal metastasis and was on treatment with oxaliplatin and fluorouracil (52). Yao et al recently presented analogous results in a 50-year-old woman with metastasis to the lungs, mediastinal lymph nodes and choroid, treated with paclitaxel, gemcitabine and adjuvant $2.5 \mathrm{mg}$ of intravitreal bevacizumab (53).

Although the use of systemic and intravitreal bevacizumab have shown promising results in case reports, one should take into account that those patients were also treated with systemic chemotherapeutic agents, that have been shown to produce reduction in choroidal metastatic lesions without the combina- tion with anti-VEGF agents (54). This raises the need to carry out more extensive trials that would better assess the benefit of this intervention.

\section{Conclusions}

Uveal metastasis is the most common malignancy of the eye $(5,9,23)$. Early detection and treatment may help avoid significant visual loss and in some cases may even aid in making the primary diagnosis. Patients with a known history of a malignancy, particularly breast or lung, who develop visual symptoms, should have a complete ophthalmic examination in search of any ocular involvement. Moreover, a multidisciplinary approach is required in those cases with a newly diagnosed uveal metastasis, as there is a significant association with the presence of central nervous system metastasis and elsewhere in the body (9).

To date, the treatment of choice for these lesions remains external beam radiotherapy that consistently produces tumor reduction and preserves vision $(22,32)$. Chemotherapeutic agents may play an important role in the future as they have been shown to be effective in the control of uveal metastasis $(20,21,23,45)$. The use of anti-VEGF therapy has become almost routine in the ophthalmology practice for many types of ocular diseases such as age-related macular degeneration, proliferative diabetic retinopathy and other ischemic diseases (55-58). Its role in the treatment of uveal metastasis remains to be determined as further reports become available in the literature. However, it may eventually help avoid the need for other more damaging and expensive treatments.

\section{References}

1. Bloch RS and Gartner S: The incidence of ocular metastatic carcinoma. Arch Ophthalmol 85: 673-675, 1971.

2. Albert DM, Zimmermann AW Jr and Zeidman I: Tumor metastasis to the eye. 3. The fate of circulating tumor cells to the eye. Am J Ophthalmol 63: 733-738, 1967.

3. Shields JA: Metastatic tumors to the uvea. Int Ophthalmol Clin 33: 155-161, 1993.

4. Wiegel T, Bottke D, Kreusel KM, et al: External beam radiotherapy of choroidal metastases - final results of a prospective study of the German Cancer Society (ARO 95-08). Radiother Oncol 64: 13-18, 2002.

5. Wiegel T, Kreusel KM, Bornfeld N, et al: Frequency of asymptomatic choroidal metastasis in patients with disseminated breast cancer: results of a prospective screening programme. $\mathrm{Br} \mathrm{J}$ Ophthalmol 82: 1159-1161, 1998.

6. Albert DM, Rubenstein RA and Scheie HG: Tumor metastasis to the eye. I. Incidence in 213 adult patients with generalized malignancy. Am J Ophthalmol 63: 723-726, 1967.

7. Kreusel KM, Wiegel T, Stange M, Bornfeld N, Hinkelbein W and Foerster MH: Choroidal metastasis in disseminated lung cancer: frequency and risk factors. Am J Ophthalmol 134: 445-447, 2002.

8. Weiss L: Analysis of the incidence of intraocular metastasis. Br J Ophthalmol 77: 149-151, 1993.

9. Shields CL, Shields JA, Gross NE, Schwartz GP and Lally SE: Survey of 520 eyes with uveal metastases. Ophthalmology 104: 1265-1276, 1997.

10. Shields JA, Shields CL and Singh AD: Metastatic neoplasms in the optic disc: the 1999 Bjerrum Lecture: part 2. Arch Ophthalmol 118: 217-224, 2000.

11. Kim CY, Ha CW and Lee SC: Vitreous and retinal metastasis from gastric cancer. Eur J Ophthalmol 20: 615-617, 2010.

12. Zografos L, Ducrey N, Beati D, et al: Metastatic melanoma in the eye and orbit. Ophthalmology 110: 2245-2256, 2003.

13. Moore RF: Choroidal sarcoma treated by the intraocular insertion of radon seeds. Br J Ophthalmol 14: 145-152, 1930. 
14. Mancini V, Battaglia M, Lucarelli G, et al: Unusual solitary metastasis of the ciliary body in renal cell carcinoma. Int $\mathbf{J}$ Urol 15: 363-365, 2008.

15. Arat $\mathrm{YO}$ and Boniuk M: Red lesions of the iris, choroid, and skin secondary to metastatic carcinoma of the thyroid: a review. Sury Ophthalmol 52: 523-528, 2007.

16. Hill VE, Brownstein S, Leonard BC and Jordan DR: Prostate adenocarcinoma presenting as a solitary choroidal metastasis. Can J Ophthalmol 33: 276-279, 1998.

17. Barsky D: Unusual tumor of the iris: a rare initial clinical manifestation of metastatic adenocarcinoma of the tail of the pancreas. Ann Ophthalmol 10: 1539-1543, 1978.

18. Zech JC, Subiger L, Chiquet C, Bouvier R and Trepsat C: Testicular choriocarcinoma metastatic to the choroid. Retina 19: $164-165,1999$.

19. Trichopoulos $\mathrm{N}$ and Augsburger JJ: Neuroendocrine tumours metastatic to the uvea: diagnosis by fine needle aspiration biopsy. Graefes Arch Clin Exp Ophthalmol 244: 524-528, 2006.

20. Demirci H, Shields CL, Chao AN and Shields JA: Uveal metastasis from breast cancer in 264 patients. Am J Ophthalmol 136: 264-271, 2003

21. D'Abbadie I, Arriagada R, Spielmann M and Le MG: Choroid metastases: clinical features and treatments in 123 patients. Cancer 98: 1232-1238, 2003.

22. Kanthan GL, Jayamohan J, Yip D and Conway RM: Management of metastatic carcinoma of the uveal tract: an evidence-based analysis. Clin Experiment Ophthalmol 35: 553-565, 2007.

23. Mewis L and Young SE: Breast carcinoma metastatic to the choroid. Analysis of 67 patients. Ophthalmology 89: 147-151, 1982.

24. Ferry AP and Font RL: Carcinoma metastatic to the eye and orbit. I. A clinicopathologic study of 227 cases. Arch Ophthalmol 92: 276-286, 1974

25. Soysal HG: Metastatic tumors of the uvea in 38 eyes. Can J Ophthalmol 42: 832-835, 2007.

26. Freedman MI and Folk JC: Metastatic tumors to the eye and orbit Patient survival and clinical characteristics. Arch Ophthalmo 105: 1215-1219, 1987.

27. Shields JA, Shields CL, Kiratli H and De Potter P: Metastatic tumors to the iris in 40 patients. Am J Ophthalmol 119: 422-430, 1995.

28. Gupta V, Gupta A and Rao NA: Intraocular tuberculosis - an update. Surv Ophthalmol 52: 561-587, 2007.

29. Papadia M, Herbort CP and Mochizuki M: Diagnosis of ocular sarcoidosis. Ocul Immunol Inflamm 18: 432-441, 2010.

30. Latendresse JR, Muskhelishvili L, Warbritton A and Tolleson WH: Two cases of uveal amelanotic melanoma in transgenic tyr-HRAS ${ }^{+}$ Ink4a/Arf heterozygous mice. Toxicol Pathol 35: 827-832, 2007.

31. Giuliari GP, Hinkle DM and Foster CS: Local treatment for lymphoid malignancies of the eye. Anticancer Agents Med Chem 9: 1123-1128, 2009.

32. Paul Chan RV and Young LH: Treatment options for metastatic tumors to the choroid. Semin Ophthalmol 20: 207-216, 2005.

33. Krema H, Somani S, Sahgal A, et al: Stereotactic radiotherapy for treatment of juxtapapillary choroidal melanoma: 3-year follow-up. Br J Ophthalmol 93: 1172-1176, 2009.

34. Egbert PR, Donaldson SS, Moazed K and Rosenthal AR: Visual results and ocular complications following radiotherapy for retinoblastoma. Arch Ophthalmol 96: 1826-1830, 1978.

35. Giuliari GP, Sadaka A, Hinkle DM and Simpson ER: Current treatments for radiation retinopathy. Acta Oncol 50: 6-13, 2011.

36. Rosset A, Zografos L, Coucke P, Monney M and Mirimanoff RO: Radiotherapy of choroidal metastases. Radiother Oncol 46 263-268, 1998

37. Tsina EK, Lane AM, Zacks DN, Munzenrider JE, Collier JM and Gragoudas ES: Treatment of metastatic tumors of the choroid with proton beam irradiation. Ophthalmology 112: 337-343, 2005.

38. Collaborative Ocular Melanoma Study Group: The COMS randomized trial of iodine 125 brachytherapy for choroidal melanoma: V. Twelve-year mortality rates and prognostic factors: COMS report no. 28. Arch Ophthalmol 124: 1684-1693, 2006.
39. Correa R, Pera J, Gomez J, et al: (125)I episcleral plaque brachytherapy in the treatment of choroidal melanoma: a singleinstitution experience in Spain. Brachytherapy 8: 290-296, 2009.

40. Pe'er J, Averbukh E and Frenkel S: Irido-cilio-choroidal melanoma in a 5 -year-old boy treated by Ru-106 brachytherapy. Clin Experiment Ophthalmol 37: 742-743, 2009.

41. Bianciotto C, Shields CL, Pirondini C, Mashayekhi A, Furuta M and Shields JA: Proliferative radiation retinopathy after plaque radiotherapy for uveal melanoma. Ophthalmology 117: 1005-1012, 2010.

42. Kiratli $\mathrm{H}$ and Bilgic S: Transpupillary thermotherapy in the management of choroidal metastases. Eur J Ophthalmol 14: 423-429, 2004.

43. Levinger S, Merin S, Seigal R and Pe'er J: Laser therapy in the management of choroidal breast tumor metastases. Ophthalmic Surg Lasers 32: 294-299, 2001.

44. Harbour JW: Photodynamic therapy for choroidal metastasis from carcinoid tumor. Am J Ophthalmol 137: 1143-1145, 2004.

45. Mewis L and Tang RA: Chemotherapy for choroidal metastases from breast carcinoma. Am J Ophthalmol 93: 810-811, 1982.

46. Piccone MR, Maguire AM, Fox KC and Gendron K: Choroidal metastases. Case 1: breast cancer. J Clin Oncol 17: 3356-3358, 1999.

47. Kosmas C, Malamos NA, Tsavaris N and Antonopoulos M: Chemotherapy-induced complete regression of choroidal metastases and subsequent isolated leptomeningeal carcinomatosis in advanced breast cancer: a case report and literature review. J Neurooncol 47: 161-165, 2000.

48. Yang JC, Haworth L, Sherry RM, et al: A randomized trial of bevacizumab, an anti-vascular endothelial growth factor antibody, for metastatic renal cancer. N Engl J Med 349: 427-434, 2003.

49. Kim SW, Kim MJ, Huh $\mathrm{K}$ and $\mathrm{Oh} \mathrm{J}$ : Complete regression of choroidal metastasis secondary to non-small cell lung cancer with intravitreal bevacizumab and oral erlotinib combination therapy. Ophthalmologica 223: 411-413, 2009.

50. Amselem L, Cervera E, Diaz-Llopis M, et al: Intravitreal bevacizumab (Avastin) for choroidal metastasis secondary to breast carcinoma: short-term follow-up. Eye (Lond) 21: 566-567, 2007.

51. Kuo IC, Haller JA, Maffrand R, Sambuelli RH and Reviglio VE Regression of a subfoveal choroidal metastasis of colorectal carcinoma after intravitreous bevacizumab treatment. Arch Ophthalmol 126: 1311-1313, 2008.

52. Lin CJ, Li KH, Hwang JF and Chen SN: The effect of intravitreal bevacizumab treatment on choroidal metastasis of colon adenocarcinoma - case report. Eye (Lond) 24: 1102-1103, 2010.

53. Yao HY, Horng CT, Chen JT and Tsai ML: Regression of choroidal metastasis secondary to breast carcinoma with adjuvant intravitreal injection of bevacizumab. Acta Ophthalmol 88: e282-283, 2010.

54. Singh A, Singh P, Sahni K, Shukla P, Shukla V and Pant NK: Non-small cell lung cancer presenting with choroidal metastasis as first sign and showing good response to chemotherapy alone: a case report. J Med Case Reports 4: 185, 2010.

55. Heier JS, Antoszyk AN,Pavan PR, et al: Ranibizumab for treatment of neovascular age-related macular degeneration: a phase I/II multicenter, controlled, multidose study. Ophthalmology 113: 633.e1, 2006.

56. Giuliari GP, Guel DA, Cortez MA and Cortez RT: Selective and pan-blockade agents in the anti-angiogenic treatment of proliferative diabetic retinopathy: a literature summary. Can J Ophthalmol 45: 501-508, 2010.

57. Giuliari GP, Guel DA and Gonzalez VH: Pegaptanib sodium for the treatment of proliferative diabetic retinopathy and diabetic macular edema. Curr Diabetes Rev 5: 33-38, 2009.

58. Michels S, Rosenfeld PJ, Puliafito CA, Marcus EN and Venkatraman AS: Systemic bevacizumab (Avastin) therapy for neovascular age-related macular degeneration twelve-week results of an uncontrolled open-label clinical study. Ophthalmology 112: $1035-1047,2005$ 